\title{
An alternative explanation of the orbital expansion of Titan and other bodies in the Solar system
}

\author{
Michal Křřžek \\ Institute of Mathematics, Czech Academy of Sciences, Žitná 25, \\ CZ-115 67 Prague, Czech Republic, e-mail: krizek@math.cas.cz \\ Vesselin G. Gueorguiev \\ Institute for Advanced Physical Studies, Sofia 1618, Bulgaria, \\ \& Ronin Institute for Independent Scholarship, 127 Haddon Pl., \\ Montclair, NJ 07043, U.S.A., e-mail: Vesselin@MailAPS.org \\ André Maeder \\ Geneva Observatory, University of Geneva, Chemin de Maillettes 51, \\ CH-1290 Sauverny, Switzerland, e-mail: andre.meader@unige.ch
}

January 12, 2022

\begin{abstract}
Recently it was found from Cassini data that the mean recession speed of Titan from Saturn is $v=11.3 \pm 2.0 \mathrm{~cm} / \mathrm{yr}$ which corresponds to a tidal quality factor of Saturn $Q \cong 100$ while the standard estimate yields $Q \geq 6 \cdot 10^{4}$. It was assumed that such a large speed $v$ is due to a resonance locking mechanism of five inner mid-sized moons of Saturn. In this paper, we show that an essential part of $v$ may come from a local Hubble expansion, where the Hubble-Lemaitre constant $H_{0}$ recalculated to the Saturn-Titan distance $D$ is $8.15 \mathrm{~cm} /(\operatorname{yr} D)$. Our hypothesis is based on many other observations showing a slight expansion of the Solar system and also of our Galaxy at a rate comparable with $H_{0}$. We demonstrate that the large disproportion in estimating the $Q$ factor can be just caused by the local expansion effect.
\end{abstract}

Keywords: local Hubble expansion; Solar system; Titan; laws of conservation of energy and angular momentum 


\section{Introduction}

There is a tendency among cosmologists to state that the Hubble expansion can only manifest itself on cosmological scales by definition. Therefore, it is believed that the Universe expands globally, but not locally. Below we show that the Universe expands at least somewhere locally.

The Hubble expansion of local systems has a long history dating back to the paper [44]. There are at least ten examples, even in the Solar system, that some tiny expansion is present. Introducing an additional effective fictitious repulsive force, to express the corresponding effects of local expansion, a number of classical paradoxes in the Solar system can be explained. For example, the Faint Young Sun Paradox (first mentioned in [10]), the very large orbital angular momentum of Titan, Triton, Charon, and our Moon, rivers on Mars, the Tidal Catastrophe Paradox of the Moon, the slow rotation of Mercury, the absence of its moons, migration of planets, etc. for details see e.g. [25, 29, 30].

The Hubble-Lemaitre constant, which describes the present expansion rate of the Universe, in century (cy) units is

$$
H_{0} \approx 70 \mathrm{~km} /(\mathrm{s} \mathrm{Mpc}) \approx 20 \mathrm{~km} /(\mathrm{s} \mathrm{Mly}) \approx 0.7 \cdot 10^{-8} \mathrm{cy}^{-1}
$$

This is the value we will adopt in the rest of the text but for general reader we would like to point out that there is about $10 \%$ variation in the value of the expansion rate depending on the type of observations utilized to deduce the value. For example, Planck Collaboration from $\mathrm{CMB}+\Lambda \mathrm{CDM}$ deduce that $H_{0} \approx 67.4 \pm 0.5 \mathrm{~km} /(\mathrm{s} \mathrm{Mpc})[52$, while Gaia + HST for Cepheids and RR Lyrae yields $H_{0} \approx 73.52 \pm 1.62 \mathrm{~km} /(\mathrm{s} \mathrm{Mpc})$ [53].

Since light travels 1 au with speed $c$ in about 500 seconds, we can recalibrate $H_{0}$ by (1) to the astronomical unit $1 \mathrm{au}=149597870700 \mathrm{~m}$ as follows

$$
H_{0} \approx \frac{0.02}{c} \mathrm{~m} \mathrm{~s}^{-1} \mathrm{yr}^{-1}=0.02 \frac{500}{\mathrm{au}} \mathrm{m} \mathrm{yr}^{-1}=10 \mathrm{~m} \mathrm{yr}^{-1} \mathrm{au}^{-1}
$$

In Section 2, we present several typical examples illustrating that this expansion rate is of the same order as some observed phenomena and measured data in the Solar system. Such an expansion rate (2) cannot be explained by the decrease of the solar mass [46], nor by the solar wind [29, p. 204], nor by tidal forces [1, p. 606]. This, of course, contradicts Kepler's laws, and thus also the law of conservation of energy, taking into account that the Solar system is sufficiently isolated from the gravitational influence of other stars. For instance, the closest star (except for our Sun) Proxima Centauri acts on the Earth with about a million times smaller gravitational force than does Venus.

The fictitious repulsive force, which appears responsible for the expansion of the Solar system and other gravitationally bounded systems, is not a new fifth physical 
force. It is only a side effect of gravitational forces. There are various possible phenomena at the origin of the observed expansion, as discussed in Section 3 . This fictitious repulsive force also influences the expansion of the Universe. Thus, there is no reason to assume that it would somehow avoid the Solar system. We also give some further arguments for the existence of a fictitious repulsive force caused by the finite speed of gravity in Section 3.1, while in Section 3.2 we mention some cosmological models [4, 15, 21, 39] that could also be at the origin of local expansion. In Section 3.3 we discuss the un-proper time parametrization view point. In Section 4 , we shall deal with a large disproportion in estimating the tidal quality factor $Q$ in the case of Saturn. Finally, Section 5 summarizes our conclusions.

\section{Typical examples of the local Hubble expan- sion - observational evidence}

In [29], a number of astrobiological, astronomical, geometrical, geochronometrical, geophysical, heliophysical, climatological, paleontological, and observational arguments is presented showing that the Solar system slowly expands by a speed of order (2). Let us briefly present some of them.

\subsection{The Earth-Moon system}

According to measurements by means of laser retroreflectors installed on the Moon, the average distance $d=384402 \mathrm{~km}$ between Earth and the Moon increases about $3.84 \mathrm{~cm} / \mathrm{yr}$, see [9]. This results in a total Hubble-like expansion per century (cy):

$$
\frac{\dot{d}}{d}=\frac{3.84 \mathrm{~cm} \mathrm{yr}^{-1}}{384402 \mathrm{~km}}=0.999 \cdot 10^{-8} \mathrm{cy}^{-1}
$$

where the dot stands for the time derivative. Only $55 \%$ of the Moon's recession of $3.84 \mathrm{~cm} / \mathrm{yr}$ can be explained by tidal forces (see [12, 24, 50, 57] for details). The missing part $v=1.72 \mathrm{~cm} \mathrm{yr}^{-1}$ could be due to the local Hubble expansion or the overall assessment of the tidal effect could be incorrect by a factor of 2. From (2) we see that this value is comparable with the average value of the Hubble-Lemaitre constant recalibrated to the distance $d=2.57 \cdot 10^{-3}$ au, namely

$$
H_{0}=1000 \mathrm{~cm} \mathrm{yr}^{-1} \mathrm{au}^{-1}=2.57 \mathrm{~cm} \mathrm{yr}^{-1} d^{-1} .
$$

The corresponding expansion rate due to fictitious repulsive force is

$$
H_{0}^{(\mathrm{loc})}=\frac{3.84(1-0.55)}{2.57} H_{0}=0.67 H_{0}
$$


A similar value was derived independently by Yurii Dumin [14,

$$
H_{0}^{(\mathrm{loc})} \approx 0.85 H_{0}
$$

Thus we see that the Earth-Moon system is expanding at significant fraction of the cosmological expansion of the Universe.

If the Earth's rotation slows down on average about $1 \mathrm{~ms}$ per century [40], then by the angular momentum conservation law of the Earth-Moon system the average distance $d$ increases about $1.25 \mathrm{~cm} / \mathrm{yr}$ using the advance of [29, pp. 184-187]. From this and the measured value $\dot{d}$ we get $3.84-1.25=2.59 \mathrm{~cm} / \mathrm{yr}$ which by $(4)$ yields for the remaining part of expansion that

$$
H_{0}^{(\mathrm{loc})} \approx H_{0}
$$

This relation also follows directly from (1) and (3).

\subsection{The Earth-Sun system}

According to Krasinsky and Brumberg [22], the present increase of the Earth-Sun distance is only $15 \mathrm{~cm}$ per year. However, their conclusion is derived under a nonrealistic supposition that the Newtonian theory of gravitation describes movements in the Solar system precisely on long-term time intervals. They solve an algebraic system for 62 unknown Keplerian elements of all planets and some large asteroids. No modeling error is assumed, no discretization error analysis is done, and the influence of a possible local expansion is not taken into account. Such small value (15 $\mathrm{cm} / \mathrm{yr}$ ) cannot result in relatively stable conditions for origin and evolution of life on Earth. The larger value (2), however, could provide the needed stable conditions for the origin and evolution of life on Earth, which has existed continually for at least 3.5 Gyr.

In $[30$, p. 61$]$, it is proved that the average recession speed

$$
v=5.2 \mathrm{~m} \mathrm{yr}^{-1}
$$

of the Earth from the Sun (cf. (2)) guarantees an almost constant solar flux on the Earth during the last (and also next) 3.5 billion years, since the luminosity of the Sun slowly and continually increases from $0.7 L_{0}$ to the present value of the solar constant, see [31,

$$
L_{0}=1.361 \mathrm{~kW} \mathrm{~m}^{-2} \text {. }
$$

To ensure favorable conditions for life on Earth it is necessary at present that variations of the luminosity to be within $5 \%$ of $L_{0}$. Note that a permanent reduction of the Sun's luminosity of more than $5 \%$ would cause an overall glaciation of the planet. 
On the other hand, at temperatures over $57^{\circ} \mathrm{C}$ DNA sequences of many multicellular organisms decay. The corresponding ring - popularly called the habitable zone (or ecosphere) - with radii $0.95^{1 / 2}$ au and $1.05^{1 / 2}$ au thus represents a very narrow interval 145.8-153.3 million $\mathrm{km}$ from the Sun. In [29, p. 216], two-sided estimates have been derived for the recession speed $v \in[4.26,6.14] \mathrm{m} / \mathrm{yr}$ guaranteeing that the luminosity changes about $5 \%$ from $L_{0}$, i.e., the Earth remains in the ecosphere during the last 3.5 Gyr.

The real mean recession speed could be even higher than $(6)$, since by data on the occurrence of fossil thermophilic bacteria [34] the temperature of oceans was about $80^{\circ} \mathrm{C}$ about $3.5 \mathrm{Gyr}$ ago. In other words, the amount of fictitious repulsive force that is continually produced by the Earth-Sun system lies in a very narrow interval that enabled the origin of life. Zhang, Lie, and Lei in their seminal paper [59] investigated solar and lunar data of hundreds of fossil patterns from the whole world. They present two other independent paleontological arguments showing that the Earth-Sun distance increases several meters per year.

\subsection{The Mars-Sun system}

In 1979, Maeder and Bouvier [38, p. 88] studied the weak field consequences of scale invariance of gravitation and suggested the possibility that the recession speed of Mars from the Sun should be several meters per year. Also by [25] and [29], Mars was closer to the Sun from 3 to 4 Gyr ago when there were rivers on its surface. At that time the luminosity of the Sun was about $0.75 L_{0}$. If Mars were to be 225 million $\mathrm{km}$ from the Sun when there was liquid water, then the corresponding solar constant for Mars would only be

$$
L_{\text {Mars }}=0.75 L_{0} \frac{150^{2}}{225^{2}}=\frac{L_{0}}{3},
$$

since the solar power decreases with the square of the distance from the Sun. The huge decrease $66.6 \%$ of luminosity as given above thus excludes the existence of rivers on Mars, if it were on the same orbit as now. Moreover, Mars had a larger albedo than now, since there were water clouds feeding hundreds of large rivers (see [29]). It is a possibility that Mars had escaped to freezing in the past thanks to a strong greenhouse effect, which could have compensated for the lower solar luminosity (7). However, the absence of carbonates in lake sediments according to analysis performed by the Curiosity Rover in Gale Crater of Mars [2] is casting serious doubts on this interpretation. For a rapid outgassing of the Martian atmosphere during the first 400 million years we refer to [55, p. 16]. We further note that if Mars were to be 180 million $\mathrm{km}$ rather than 225 million $\mathrm{km}$ from the Sun when it originated, then its average recession speed would be $10 \mathrm{~m} / \mathrm{yr}$ which is again comparable to the value of the Hubble-Lemaître constant as given in (2). 


\subsection{The Saturn system and beyond}

According to two independent astrometric and radiometric Cassini data [32, 33, the mean recession speed of Titan from Saturn is

$$
v \approx 11.3 \mathrm{~cm} / \mathrm{yr}
$$

Before these measurements, astronomers assumed that this speed is only $0.1 \mathrm{~cm} / \mathrm{yr}$, because Saturn is mostly a gaseous planet and thus tidal forces are small. (Note that oceanic tides play a predominant role in the secular deceleration of the Earth's rotation, see [51].) Since the Titan-Saturn average distance is $D=1221870 \mathrm{~km}$, the value (8) results in:

$$
\frac{\dot{D}}{D}=\frac{11.3 \mathrm{~cm} / \mathrm{yr}}{1221870 \mathrm{~km}}=0.925 \cdot 10^{-8} \mathrm{cy}^{-1},
$$

by means of (2) the recalibrated value of the Hubble-Lemaitre constant is

$$
H_{0}=8.15 \mathrm{~cm} \mathrm{yr}^{-1} D^{-1} \text {. }
$$

Then the corresponding expansion rate is

$$
H_{0}^{(\mathrm{loc})}=\frac{11.3}{8.15} H_{0}=1.38 H_{0} .
$$

This value, of course, includes tides which have to be subtracted like in the case of our Moon, see (5). Moreover, resonances with mid-sized inner moons should also be taken into account.

Other satellites of planets are also affected by fictitious repulsive forces. In particular, there exist 11 fast satellites that are below the stationary orbit of Uranus, where the orbital and rotational periods are equal. These satellites should approach their mother planet along spiral trajectories due to tidal forces. However, a fictitious repulsive force acts in the opposite direction and has approximately the same size as tidal forces. This makes trajectories of fast satellites stable for billions of years (see $[29$, p. 227] for details). The fictitious repulsive forces, thus, prevent all fast satellites from crashing into their mother planets.

It is an open problem how Neptune could be formed as far away as 30 au from the Sun, where the original protoplanetary disc was relatively sparse and all motions are very slow (by Kepler's third law its mean speed is only $5.4 \mathrm{~km} / \mathrm{s}$ ). Assuming that the average Neptune-Sun distance increases roughly at the rate comparable with (2), we find (as in previous examples) that Neptune could be formed about 10 au closer to the Sun 4.5 Gyr ago and then migrate due to the fictitious repulsive force to its actual orbit. Resonances with Jovian planets could, of course, also play an essential role in this process. 
Let us present several more examples. There is a well-known puzzle, how the retrograde moon Triton was captured so far from Neptune on almost circular orbit with large radius $a=354759 \mathrm{~km}$. We claim that Triton was captured closer and then migrated from the gaseous planet Neptune, since the fictitious repulsive force can be bigger than tidal forces that act in opposite direction. This could explain the present very large Triton's orbital angular momentum.

Similar arguments can be applied to the Pluto-Charon system. Since this system is completely locked into the 1:1:1 resonance, tidal forces are negligible and its large orbital momentum can again be due to the fictitious repulsive force. For more information about the local Hubble expansion we refer to [12, 14, 25, 26, 27, 28, 30, 36. [37. 38, 39, 40].

In [29], several arguments are presented showing that also galaxies themselves slightly expand at rate comparable with $H_{0}$. For instance, by [41, Sect. 8] the observed conservative expansion rate of the Milky Way is $0.6-1 \mathrm{kpc} / \mathrm{Gyr}$, which is approximately $0.6-1 \mathrm{~km} / \mathrm{s}$, since $1 \mathrm{kpc}=3.086 \cdot 10^{16} \mathrm{~km}$ and $1 \mathrm{Gyr}=3.156 \cdot 10^{16} \mathrm{~s}$. This expansion rate nicely fits to the Hubble constant recalculated by (1) on the radius $R=50000$ ly $=15328$ pc of our Galaxy, namely, $H_{0}=1 \mathrm{~km} /(\mathrm{s} R)$ and

$$
\frac{\dot{R}}{R}=\frac{1 \mathrm{kpc} / \mathrm{Gyr}}{15328 \mathrm{pc}}=0.652 \cdot 10^{-8} \mathrm{cy}^{-1} \text {. }
$$

This value is again comparable with similar ratios (3) and (9) which include effects of tidal forces. Some of the above arguments are summarized in Table 1. Further arguments are given in Section 3.

\begin{tabular}{|l|c|l|}
\hline system & $H_{0}^{(\text {loc })} / H_{0}$ & references \\
\hline Earth-Moon & $0.5-1^{*}$ & {$[12,14,24]$} \\
Sun-Earth & $0.5-1$ & {$[25,29,59]$} \\
Sun-Mars & $0.3-1$ & {$[25,38]$} \\
Saturn-Titan & $\approx 1.3^{*}$ & {$[32,33]$} \\
Sun-Neptune & $\approx 1$ & {$[29,30]$} \\
Galaxy & $0.6-1^{*}$ & {$[29,41]$} \\
\hline
\end{tabular}

Table 1: Approximate relative values of the observed local Hubble expansion for various systems. The values with stars are based on direct measurements/observations related to the Earth-Moon system, Saturn-Titan system, and our Galaxy, while the rest are indirectly deduced. 


\section{The origin of the local Hubble expansion}

The validity of physical laws is verified by measurements. Nevertheless, absolutely exact measurement instruments cannot be constructed. Hence, we are not able to check by measurements whether generally accepted physical laws are valid for an arbitrary number of decimal digits. For instance, the law of conservation of energy belongs to the basic pillars of current physics. However, is this law valid also in the Solar system or in our Galaxy that are only modeled by Newton's theory?

The Newtonian gravitational law represents only a certain idealization of reality. It yields reliable results on short time intervals and should not necessarily be applied on long term intervals due to large modeling errors. The Newtonian theory of gravitation is formulated so that the laws of conservation of energy and angular momentum are valid absolutely exactly. Anyway, it does not satisfy the Principle of causality because of infinite speed of gravity, which causes that gravitational interaction immediately gets outside the corresponding light cone. However, the Principle of causality should have a priority to the laws of conservation of energy and angular momentum.

\subsection{Gravitational aberration considerations}

Retardation effects in the theory of gravity were considered already in the 1898 paper [18] by Paul Gerber. Nevertheless, at present they are usually neglected in astrophysical modeling, see e.g. [58. Another approach which satisfies the Principle of Causality and which does not satisfy the laws of conservation of energy and angular momentum was first introduced by Sir Arthur Eddington in [16, p. 94]. To explain his main idea, consider for simplicity only two bodies $A$ and $B$ of equal point masses $m_{A}=m_{B}>0$ that orbit symmetrically with respect to their center of gravity. If $A$ attracts $B$ and $B$ also attracts $A$ at their instantaneous positions, then by the Newtonian theory of gravitation these forces are in the same line and in balance.

However, the speed of gravitational interaction is finite, actually $c_{\mathrm{g}}=c$. When the body $A$ is in $A^{\prime}$, it feels the attraction from $B$ (instead of $B^{\prime}$ ) due to time delay as depicted in Fig. 1. Similarly $B^{\prime}$ feels the attraction from $A$. Thus, the centripetal force is stronger by the factor $1 / \cos ^{2} \alpha$, where $\alpha=A B A^{\prime}=B A B^{\prime}$ are gravitational aberration angles. This favors an overall contraction of the system. Nevertheless, there is a tangential forward component of the force, since $B$ is less "backward" than $B$ ' as seen from $A$. Then a couple of very small non-equilibrium tangential forces arise that permanently act on this post-Newtonian system. This seems to result in increase of the total angular momentum and the total energy of the system. Hence, the corresponding trajectories form two very slowly expanding spirals (see Fig. 1).

Emmy Noether was a leading mathematician who proved the following theorem [47], which completely changed the face of physics: The energy of each isolated system 
is conserved if it possesses symmetry with respect to time translations. Nonetheless, this symmetry is not true for spiraling trajectories caused by fictitious repulsive force. Similarly, another Noether's theorem states that symmetry under rotation, or rotational invariance, leads to the conservation of angular momentum. This symmetry is also slightly violated in our case.

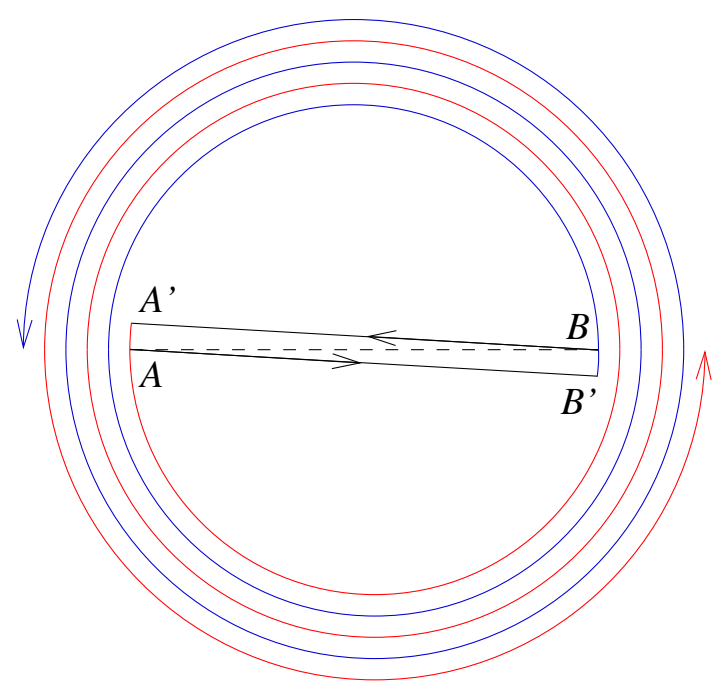

Figure 1: Schematic illustration of trajectories of two interacting bodies of equal masses that form a double spiral. The gravitational aberration angles $\alpha=A B A^{\prime}$ and $\alpha=B A B^{\prime}$ are extremely small but positive.

To see how non-conservative forces could arise in the above model, notice that the retarded gravitational force $F_{A B^{\prime}}$ is at aberration angle $\alpha$. Thus, resulting in force $F_{A B^{\prime}} \times \cos \alpha \approx F_{A B}(\cos \alpha)^{-2} \cos \alpha$ which is larger by a factor $1 / \cos \alpha$ than the usually considered force $F_{A B}$ along the line $A B$ that goes through the center of mass of the system. Thus, the system is not in the usual equilibrium of gravitational and centrifugal forces $\left(F_{A}=m_{A} R_{A} \omega_{A}^{2}\right)$. Furthermore, the forward pushing tangential force $F_{\mathrm{t}}=F_{A B^{\prime}} \times \sin \alpha$ is proportional to the orbital speed $v$, since the aberration angle $\alpha \approx \tan \alpha=v / c$. The presence of velocity-dependent force $F_{\mathrm{t}} \propto v$ results in a dissipative-like energy effect due to the non-conservative nature of such force term. This velocity-dependent term could also induce angular momentum non-conservation, and as a result possible adjustment of Kepler's third law.

The key observation here is the presence of an additional force component along the path of the object with a magnitude $F_{\mathrm{t}} \approx F_{A B^{\prime}} \times \frac{v}{c}$. The corresponding instantaneous additional acceleration is then expected to be $a_{\mathrm{t}} \approx \frac{F_{A B}}{\gamma^{2} m_{B}} \frac{v}{c}$, where $\cos \alpha \approx$ 
$\gamma=\sqrt{1-(v / c)^{2}}$. Note that when expanded in powers of $v / c$ the force effect is of 3rd order in $v / c$. If this additional acceleration is assumed to be the fictitious acceleration due to un-proper time parametrization, see equation (21) of [20], then for a two-body system, like the one shown in Fig. 1, we can estimate the value of the proportionality parameter $\kappa_{0}$ in $a_{\mathrm{t}}=\kappa_{0} v$ to be $\kappa_{0} \approx G \frac{M}{\gamma^{2} R^{2} c}$. When applied to the Earth-Moon system the result is $G \frac{M}{\gamma^{2} R^{2} c} \approx 0.0287 \mathrm{cy}^{-1}$. This number is too big in magnitude to have escaped detection via the observed Lunar recession that suggests many orders of magnitude smaller value for $\kappa_{0}$ based on the $\dot{d} / d$ value in (3). Therefore, such gravitational aberration at third order in $v / c$ may not be actually present as discussed by Carlip [5]. On other hand, the gravitational radiation effect is much too small since it is of fifth order in $v / c \approx 3.5 \cdot 10^{-6}$; that is, $G \frac{M}{\gamma^{2} R^{2} c}(v / c)^{2} \approx 3.3 \cdot 10^{-13} \mathrm{cy}^{-1}$.

The above discussion seems to lead the gravitational aberration idea into a dead alley. However, if one embraces the ideas developed below by looking at the energy dissipated by the force $F_{\mathrm{t}}$, then one has $\dot{E}=F_{\mathrm{t}} v=G \frac{M m v^{2}}{\gamma^{2} R^{2} c}=726 \mathrm{PW}$, while the overall energy will be approximated by $E \approx E_{1}+E_{2}+U \approx 1.75 \cdot 10^{29} \mathrm{~J}$ neglecting the Moon rotational energy (this notation is introduced below). Therefore, for the Earth-Moon system this results in $\dot{E} / E \approx 1.3 \cdot 10^{-4} \mathrm{yr}^{-1}$ which per Lunar period $T=27.32$ days gives the dimensionless number $T \dot{E} / E \approx 9.8 \cdot 10^{-6}$. These numbers are sufficiently small to evade detection so far but big enough to be non-negligible compared to the gravitational radiation effect as discussed in the previous paragraph.

In the above estimates we have used that the Earth moment of inertia is $I_{1}=$ $8.036 \cdot 10^{37} \mathrm{~kg} \mathrm{~m}^{2}$ (see [3], also [29, p. 183]) and the corresponding rotational angular frequency is $\omega_{1}=7.292 \cdot 10^{-5} \mathrm{~s}^{-1}$. Hence, the Earth rotational momentum is

$$
L_{1}=I_{1} \omega_{1}=5.86 \cdot 10^{33} \mathrm{~kg} \mathrm{~m}^{2} / \mathrm{s}
$$

and its rotational energy is $E_{1} \approx \frac{1}{2} L_{1} \omega_{1}=2.125 \cdot 10^{29} \mathrm{~J}$. The orbital momentum of the Moon is

$$
L_{2}=m_{2} d^{2} \Omega_{2}=2.89 \cdot 10^{34} \mathrm{~kg} \mathrm{~m}^{2} / \mathrm{s},
$$

where $m_{2}=7.3477 \cdot 10^{22} \mathrm{~kg}$ is the Moon's mass, $\Omega_{2}=2 \pi / T=2.662 \cdot 10^{-6} \mathrm{~s}^{-1}$ is the orbital angular velocity. The Moon orbital energy is thus $E_{2} \approx \frac{1}{2} L_{2} \Omega_{2}=0.385 \cdot 10^{29} \mathrm{~J}$. The corresponding gravitational potential energy is $U=-G m_{1} m_{2} / d=-0.762 \cdot 10^{29} \mathrm{~J}$, where $m_{1}=5.9722 \cdot 10^{24} \mathrm{~kg}$ is the Earth's mass.

In the framework of Special and General Relativity Carlip derived that retardation effects resulting from velocity dependent field potentials give rise to forces that are linear (for electromagnetism) and quadratic (for gravity) extrapolations pointing towards the instantaneous source location [5]. He showed that "Although gravity propagates at the speed of light in general relativity, the expected aberration is almost exactly canceled by velocity-dependent terms in the interaction". Note that this remarkable result is about the force on a test particle that does not alter (by 
definition) the overall field produced by the source. Furthermore, Carlip argues that aberration effects become significant only at powers consistent with radiation effects that are expected at order $v^{3} / c^{3}$ for electromagnetism and $v^{5} / c^{5}$ for gravity. However, if one assumes that the laws of conservation of energy and angular momentum hold exactly, then one cannot get spiraling trajectories as in Fig. 1.

Note that the energy dissipated by the force $F_{\mathrm{t}}$ discussed in the previous paragraph can also be written in terms of the gravitational potential energy $F_{\mathrm{t}} v=-U \times\left(\frac{v}{c}\right) \frac{v}{\gamma R}$. It can also be viewed as energy dissipated by the receding speed against the gravitational force $F_{\mathrm{t}} v=F_{A B^{\prime}} v^{2} / c=F_{A B^{\prime}} v_{r}$. This provides an estimate of the local Hubble expansion $H_{0}^{(\text {loc })}=v_{r} / r \approx \frac{v^{2}}{c r}=\left|v F_{\mathrm{t}} / U\right| \approx 0.03 \mathrm{cy}^{-1}$, which seems to be missing a factor $v / c$ to be almost the same order of magnitude with (1). This suggests that following Carlip's approach and computing exactly the high-order terms in $v / c$ beyond the first few orders can be very informative in understanding the origin and level of contribution of the aberration effects.

It has been discussed that gravitational aberration is associated with very small fictitious repulsive force [24]. The gravitational aberration effects can be modeled by a non-autonomous system of differential equations with delays. However, the simplest post-Newtonian model produces relatively quickly expanding trajectories. The reason is that it does not take into account a curved space-time (nor gravitational waves). The proper distance between point masses $m_{A}$ and $m_{B}$ is, in fact, larger than the distance of their projection into the flat coordinate Euclidean space. This mechanism contributes to the local Hubble expansion which seems to lead to small discrepancies from exact conservation of energy and angular momentum within the Solar system. This hypothesis even has the potential to substantially explain the dark energy problem [30, 54].

\subsection{Cosmic considerations}

In order to estimate the possible magnitude of such effects we now discuss the series expansion of the scale factor $a(t)$ in terms of Hubble parameter $H(t)$ and deceleration parameter $q(t)$. The Hubble parameter is defined by the well-known relation

$$
H=\frac{\dot{a}}{a}
$$

where $a$ is the expansion function and the dot denotes its time derivative. Differentiating $H=H(t)$ with respect to time, we find that

$$
\dot{H}=\frac{\ddot{a}}{a}-H^{2}=-q H^{2}-H^{2},
$$


where $q=-\ddot{a} a / \dot{a}^{2}$ is the deceleration parameter. The Taylor expansion reads (see e.g. [45, p. 781], [49, p. 313])

$$
a(t)=a\left(t_{0}\right)\left(1+H_{0} \Delta t-\frac{1}{2} q_{0} H_{0}^{2}(\Delta t)^{2}+\ldots\right),
$$

where $t_{0}$ is the present time, $H_{0}=H\left(t_{0}\right), \Delta t=t-t_{0}$, and $q_{0}=q\left(t_{0}\right) \approx-0.6$ is the actual value of the deceleration parameter.

An extremely small time derivative of $H(t)$ for the present time on the scale of the Solar system is derived in [6] yielding a tiny outward acceleration of $2 \cdot 10^{-23} \mathrm{~m} / \mathrm{s}^{2}$ at Pluto's distance of $40 \mathrm{au}$. Similar very small values are given in papers [7, p.62], [8, p. 435], and [42, p. 5041] claiming that the cosmological constant $\Lambda$ has negligible influence on the Solar system. These papers, in fact, state that the quadratic term in the above Taylor expansion has almost no effect on the accelerated expansion of the Solar system which is true, but surprisingly they do not take into account the large value of the Hubble-Lemaître constant itself (see (2)) which appears at the linear term in 16 . For instance, if $\Delta t=1 \mathrm{yr}$, then by (2) we obtain

$$
H_{0} \Delta t=10 \mathrm{~m} / \mathrm{au}=0.668 \cdot 10^{-10} .
$$

Hence, the linear term is much larger than the quadratic term in (16),

$$
\left|H_{0} \Delta t\right| \gg \frac{1}{2}\left|q_{0}\right| H_{0}^{2}(\Delta t)^{2}
$$

for $\Delta t$ close to 0 . Consequently, the accelerated expansion is nonnegligible only at cosmological distances due to (18). However, if $|\Delta t|=10$ Gyr, then by (17) we still have

$$
\left|H_{0} \Delta t\right|=0.668>0.134=\frac{1}{2}\left|q_{0}\right| H_{0}^{2}(\Delta t)^{2},
$$

i.e., the linear term in (16) essentially dominates over the quadratic term not only on small time scales but also at time scales of the order of the age of the Solar system and the Milky Way galaxy.

Thus, on the whole we see that there are various physical effects through which some local expansion could be generated. The observation of such effects will need years of data and perhaps even centuries of accumulation of such small trends to become noticeable.

\subsection{Non-conservation effects}

In this section we discuss how non-conservation effects could be due to the use of unproper time parametrization and can be controlled by a parameter $\kappa_{0}$. The un-proper time parametrization leads to fictitious acceleration proportional to the velocity of a 
body of the form $\kappa_{0} \vec{v}[20]$. Similar acceleration term is also present in the week field limit of the SIV theory [38, 39]. (For another reason see also [13].) The value of $\kappa_{0}$ was suggested to be related to the aberration effects earlier in the paper (see Section 3.1 ), however, the first possibility $G \frac{M}{\gamma^{2} R^{2} c} \approx 0.0287 \mathrm{cy}^{-1}$, which is at the $3 \mathrm{rd}$ order of $v / c$, could possibly be relatively too large, while the gravitational radiation effect is too small, since it is at the 5 th order of $v / c$. Nevertheless, tidal effects and random impacts do affect the parts of the particular planetary system. For example, in the Earth-Moon system the leading effect is the loss of rotational kinetic energy $E \propto \mathcal{I} \omega^{2}$ (see $(23)$ of $[20]$ ) that results in $\dot{E} / E \approx 2 \dot{\omega} / \omega=-2 \dot{T} / T$, where $\omega$ is the Earth rotational rate $2 \pi / T$. By (13) and (14) the leading term in the angular momentum (see (24) of [20]) is the Moon orbital momentum $L_{2}=27.92 \cdot 10^{33} \mathrm{~kg} \mathrm{~m}^{2} / \mathrm{s}$ which is a factor of 4.8 bigger than Earth rotation momentum $L_{1}=5.86 \cdot 10^{33} \mathrm{~kg} \mathrm{~m}^{2} / \mathrm{s}$. Thus, one has $\dot{L} \approx 2 L_{2} \dot{d} / d+L_{1} \dot{\omega} / \omega+\ldots$ If one insists on the usual angular momentum conservation argument then one obtains

$$
\frac{\dot{d}}{d} \approx-\frac{L_{1}}{2 L_{2}} \frac{\dot{\omega}}{\omega}=-\frac{5.86}{2 \cdot 27.92} \frac{\dot{\omega}}{\omega}=0.1 \frac{\dot{T}}{T} .
$$

When using $\frac{\dot{\omega}}{\omega}=-\frac{\dot{T}}{T}$ with length of the day change per century $\dot{T} \approx 1 \mathrm{~ms} / \mathrm{cy}$ [40] and the standard length of the Earth sidereal day $(T=86164.1 \mathrm{~s})$, which is resulting in $\dot{T} / T=1.157 \cdot 10^{-8} \mathrm{cy}^{-1}$, one arrives at almost an order of magnitude $\approx 8.6$ times smaller value for the Moon's recession rate than the experimentally observed rate (3):

$$
\frac{\dot{d}}{d} \approx 0.1 \frac{1 \mathrm{~ms} / \mathrm{cy}}{T}=0.1 \frac{1 \mathrm{~ms} / \mathrm{cy}}{86164.1 \mathrm{~s}}=0.116 \cdot 10^{-8} \mathrm{cy}^{-1}
$$

If we apply energy conservation: $0=\dot{E}=2 E_{2} \dot{d} / d-U \dot{d} / d+2 E_{1} \dot{\omega} / \omega+\ldots$ then one would have a difficult constraint to satisfy $\dot{d} / d \approx-2 E_{1} /\left(2 E_{2}-U\right)(\dot{\omega} / \omega)$ that implies $2 E_{1} /\left(2 E_{2}-U\right) \approx L_{1} /\left(2 L_{2}\right)$ which is not satisfied for the Earth-Moon system.

If one considers non-conservation, as discussed in sub-section 3.1, using $L=L_{1}+L_{2}$ and $E=E_{1}+E_{2}+U$ along with the Virial theorem $\left\langle U>\approx-2<E_{2}\right\rangle$, then one has

$$
\begin{gathered}
\dot{E} \approx 2 E_{1} \frac{\dot{\omega}}{\omega}+2 E_{2} \frac{\dot{d}}{d}-U \frac{\dot{d}}{d}+\cdots \Rightarrow \dot{E} \approx 2 E_{1} \frac{\dot{\omega}}{\omega}+4 E_{2} \frac{\dot{d}}{d}+\ldots \\
\kappa_{0}=\frac{\dot{L}}{L} \approx 2 \frac{L_{2}}{L} \frac{\dot{d}}{d}+\frac{L_{1}}{L} \frac{\dot{\omega}}{\omega}+\ldots
\end{gathered}
$$

By eliminating $\kappa_{0}$ we obtain 1

$$
2 \frac{L_{2}}{L} \frac{\dot{d}}{d}+\frac{L_{1}}{L} \frac{\dot{\omega}}{\omega} \approx-\frac{4 E_{2}}{E} \frac{\dot{d}}{d}-2 \frac{E_{1}}{E} \frac{\dot{\omega}}{\omega} .
$$

\footnotetext{
${ }^{1}$ We have to use $\dot{E} / E=-\kappa_{0}$ in order to agree with the loss of mechanical energy and assuming $\kappa_{0}>0$.
} 
Which can further be approximated using $E \approx E_{1}$ and $L \approx L_{2}$ :

$$
2 \frac{\dot{d}}{d}+\frac{L_{1}}{L_{2}} \frac{\dot{\omega}}{\omega} \approx-\frac{4 E_{2}}{E_{1}} \frac{\dot{d}}{d}-2 \frac{\dot{\omega}}{\omega}
$$

which simplifies to

$$
\frac{\dot{d}}{d} \approx-\frac{\left(2 L_{2}+L_{1}\right) E_{1}}{2\left(E_{1}+2 E_{2}\right) L_{2}} \frac{\dot{\omega}}{\omega} \approx-0.8197 \frac{\dot{\omega}}{\omega} \approx 0.948 \cdot 10^{-8} \mathrm{cy}^{-1} .
$$

Here we have used again $\dot{T} / T=1.157 \cdot 10^{-8} \mathrm{cy}^{-1}$. This value is much closer to the measured value (3) then one based on conservation of the angular momentum (19).

The Solar system and our Galaxy provide us with a unique laboratory to study whether the laws of conservation of energy and angular momentum are valid, see e.g. $[29,48]$. The fictitious forces cause secular migration of planets and their moons of order (2). The observed slight expansion of the Solar system indicates that the laws of conservation of energy and angular momentum are slightly violated.

\section{A large disproportion in estimating the ef- fective tidal quality factor}

From Table 2 we see that Titan and five mid-sized inner moons of Saturn have almost circular orbits and small inclinations with respect to Saturn's equator (see e.g. [11, [17, 56]). All these moons have synchronous rotation, i.e., there is 1:1 resonance between each moon's rotation and its orbital period around Saturn. From Table 2 we further observe that the orbital angular momentum of Rhea is only $1.1 \%$ of Titan's momentum and the orbital angular momenta of other inner moons are much smaller. The radius of Titan's orbit is quite large with respect to orbits of other moons so that the resonance effects (like 4:2:1 resonance of Io, Europa and Ganymede, see [17]) have to be small.

Tidal bulges raised by planets and their moons are characterized by the effective tidal quality dimensionless factor $Q:=1 /|2 \tan \theta| \approx 1 /|2 \theta|$, where $\theta$ is the tidal lag angle, see [35]. For instance, if moon's revolution period is larger than planet's rotation period, then the lagging tide is carried ahead of the moon by the angle $\theta>0$. On the other hand, moons below the stationary orbit produce a negative angle $\theta<0$. For bodies on the stationary orbit, the factor $Q$ is not well defined.

According to [19], real values of $Q$ separate sharply into two groups. Values in the range 10 to 500 correspond to the terrestrial planets and all moons with $\theta \neq 0$ while values

$$
Q \geq 6 \cdot 10^{4}
$$




\begin{tabular}{|l|c|r|r|r|r|r|}
\hline Moon & $m(\mathrm{~kg})$ & $a(\mathrm{~km})$ & $e$ & $i\left(^{\circ}\right)$ & $\bar{v}(\mathrm{~km} / \mathrm{s})$ & $L\left(\mathrm{~kg} \mathrm{~m}^{2} / \mathrm{s}\right)$ \\
\hline Mimas & $3.7493 \cdot 10^{19}$ & 185539 & 0.0196 & 1.574 & 14.28 & $9.934 \cdot 10^{19}$ \\
Enceladus & $1.0802 \cdot 10^{20}$ & 237948 & 0.0047 & 0.009 & 12.69 & $3.262 \cdot 10^{20}$ \\
Tethys & $6.1745 \cdot 10^{20}$ & 294619 & 0.0001 & 1.120 & 11.35 & $2.065 \cdot 10^{21}$ \\
Dione & $1.0955 \cdot 10^{21}$ & 377396 & 0.0022 & 0.019 & 10.03 & $4.147 \cdot 10^{21}$ \\
Rhea & $2.3065 \cdot 10^{21}$ & 527108 & 0.0013 & 0.345 & 8.48 & $1.031 \cdot 10^{22}$ \\
Titan & $1.3452 \cdot 10^{23}$ & 1221870 & 0.0288 & 0.349 & 5.57 & $9.155 \cdot 10^{23}$ \\
\hline
\end{tabular}

Table 2: Basic parameters of the relevant Saturn's moons, where $a$ is the semi-major axis, $e$ is the eccentricity, $i$ is the inclination to Saturn's equator, $\bar{v}$ is the mean orbital speed, and $L=m a \bar{v}$ is the orbital angular momentum.

correspond to the major planets. The associated tidal lag angles fulfill $|\theta| \in\left(0.06^{\circ}, 3^{\circ}\right)$ and $|\theta| \leq 1.7^{\prime \prime}$, respectively.

A key problem is to estimate the factor $Q$ corresponding to Saturn, where the reciprocal value $1 / Q$ is proportional to the thermal dissipation exerted by Titan and other moons in the interior of Saturn. According to [17, 32, 33], one has

$$
\frac{\dot{a}}{a}=\frac{3 k_{2}}{Q} \frac{M_{\text {moon }}}{M_{\mathrm{S}}}\left(\frac{R_{\mathrm{S}}}{a}\right)^{5} \Omega=: \frac{b}{Q}
$$

where $M_{\text {moon }}$ is the mass of the moon, $R_{\mathrm{S}}=58232 \mathrm{~km}$ and $M_{\mathrm{S}}=5.6834 \cdot 10^{26} \mathrm{~kg}$ are the mean radius and mass of Saturn, respectively, $\Omega=2 \pi / T$ is the moon's mean motion, $T=15.945$ days is the orbital period of Titan, and $k_{2} \approx \frac{1}{3}$ is the dimensionless Love number of degree two which describes the rigidity and surface deformations of Saturn under a tidal potential.

If one is to use (20) in (21) for Titan, then its recession speed is estimated to be $\dot{a}<0.2 \mathrm{~cm} / \mathrm{yr}$, which is less than $2 \%$ of the observed recession (8). Alternatively, if one estimates $Q$ by using the Cassini measurements (8), Table 2, and the left-hand side of (21) for Titan as given by (9) - the result is $Q=90.5$. However, the theory of major planets [43, p. 587] predicts much larger values for $Q$ satisfying (20).

Lainey et al. $[32,33]$ explain this extremely large disproportion by resonance locking of five inner mid-sized moons from Table 2. They consider a complex Love number and perform a Monte-Carlo approach. The basic idea of tidal migration via resonances was presented earlier in [17]. There is a 2:1 resonance between Mimas and Tethys and also between Enceladus and Dione, but no resonance between Titan and the other moons.

We propose another explanation that could essentially help in solving the above controversial issue. If the total Titan recession (8) is mostly due to local Hubble 
expansion (10), then the tidal effects contribute only $11.3-8.15=3.15 \mathrm{~cm} / \mathrm{yr}$. Since $1 \mathrm{yr}=31558149.54 \mathrm{~s}$, the corresponding estimate of the $Q$ factor is by (21)

$$
Q=\frac{b D}{3.15} \approx 325
$$

We see that this value is still a few orders of magnitude smaller than the expected value (20). It suggests that recession due to the tidal effects and local Hubble expansion are not simply additive contributions to the overall recession process. In fact, Fig. 2 of [32] is effectively a Hubble diagram showing a local expansion with a clear HubbleLemaitre constant. Therefore, the local Hubble expansion provides an alternative argument for explaining the large disproportion in estimating the effective tidal quality factor for Saturn.

\section{Conclusions}

The main point of this work is to emphasize the overall convergence of the observed orbital expansion of different systems such as the Earth-Moon, Titan-Saturn with the general Hubble cosmological expansion. The past history of the Martian climate, with many evidences of liquid water connected to the relative absence of carbonates, is also consistent with an expansion of the Martian orbit at the same rate. Of course, each of these systems results from complex dynamical and astrophysical processes, however despite the differences of these systems, the similarity of the effects and of their relative sizes is striking.

As to the origin of the observed expansion, we have examined a number of effects and their order of magnitude in the framework of General Relativity, and also by considering effects due to fictitious acceleration either aberrational or due to unproper time parametrization. The problems found locally in the Solar system may also be related to the dark components of the Universe, so that different cosmological models $[39,4]$ could also possibly be at the origin of the observed local expansion. For instance, according to [13] and [15], the local Hubble expansion arises in a specific cosmological asymptotics of the Robertson-Walker metric at infinity (cf. also [21]).

The fictitious forces cause secular migration of planets and their moons of order (2). The observed slight expansion of the Solar system indicates that the laws of conservation of energy and angular momentum are slightly violated. If this force were not to act in the Solar system, then conditions favorable for the development of life on Earth would exist for only about 1 Gyr. Intelligent life would not have had enough time to develop due to a continual rise of temperature on Earth, see [25, 30].

Acknowledgment. The authors thank Filip Kř́žek, Jaroslav Pavlousek, and Weijia Zhang for fruitful discussions. M. Křrižek was supported by the Institute of Math- 
ematics of the Czech Academy of Sciences (RVO 67985840). V. Gueorguiev is extremely grateful to his wife and daughters for their understanding and family support. A. Maeder expresses his deep gratitude to his wife and to D. Gachet for their continuous support.

\section{References}

[1] B. Bertotti, P. Farinella, D. Vokrouhlický, Physics of the Solar system, Kluwer, Dordrecht, 2003.

[2] T.F. Bristow, R. M. Haeberle, D. F. Blake et al., "Low Hesperian PCO2 constraints from in situ mineralogical analysis of Gale Crater, Mars," Proc. Nat. Acad. Sci. 114, 2166-2170 (2017).

[3] M. Burša, K. Peč, Gravity field and dynamics of the Earth, Springer, Berlin, 1993.

[4] S. Capozziello, C. Stornaiolo, "Space-time deformations as extended conformal transformations," Int. J. Geom. Meth. Mod. Phys. 5, 185-195 (2008).

[5] S. Carlip, "Aberration and the speed of gravity," Phys. Lett. A 267, 81-87 (2000).

[6] M. Carrera, D. Giulini, "Influence of global cosmological expansion on local dynamics and kinematics," Rev. Mod. Phys. 82, 169-208 (2010).

[7] F. I. Cooperstock, V. Faraoni, D. N. Vollick, "The influence of the cosmological expansion on local systems," Astrophys. J. 503, 61-66 (1998).

[8] R. H. Dicke, P. J.E. Peebles, "Evolution of the Solar system and the expansion of the universe," Phys. Rev. Lett. 12, 435-437 (1964).

[9] J. O. Dickey et al., "Lunar laser ranging: A continuing legacy of the Apollo program," Science 265, 482-490 (1994).

[10] W. L. Donn, B. D. Donn, W. G. Valentine, "On the early history of the Earth," Bull. Geol. Soc. Amer. 76, 287-306 (1965).

[11] M.K. Dougherty, L. W. Esposito, S. M. Krimigis (eds.), Saturn from CassiniHuygens, Springer, Dordrecht, 2009.

[12] Y.V. Dumin, A new application of the Lunar laser retroreflectors: "Searching for the "local" Hubble expansion," Adv. Space Res. 31, 2461-2466 (2003).

[13] Y. V. Dumin, "Comment on 'progress in lunar laser ranging tests of relativistic gravity'," Phys. Rev. Lett. 98, 059001 (2007). 
[14] Y.V. Dumin, "Testing the dark-energy-dominated cosmology by the SolarSystem experiments," Proc. of the 11th Marcel Grossmann Meeting on General Relativity (eds. H. Kleinert, R. T. Jantzen, R. Ruffini), World Sci., Singapore, 1752-1754, 2008.

[15] Y. V. Dumin, "Lambda perturbations of Keplerian orbits in the expanding Universe," Grav. Cosmol. 26, 307-315 (2020).

[16] A. Eddington, Space, time and gravitation, Cambridge Univ. Press, Cambridge, 1966.

[17] J. Fuller, J. Luan, E. Quataert, "Resonance locking as the source of rapid tidal migration in the Jupiter and Saturn moon systems," Mon. Not. R. Astron. Soc. 458, 3867-3879 (2016).

[18] P. Gerber, "Die räumliche und zeitliche Ausbreitung der Gravitation," Zeitschrift Math. Phys. 43, 93-104 (1898).

[19] P. Goldreich, S. Soter, "Q in the Solar System," Icarus 5, 375-389 (1966).

[20] V.G. Gueorguiev, A. Maeder, "Geometric justification of the fundamental interaction fields for the classical long-range forces," Symmetry 13, 379, 24 pp. (2021); doi:10.3390/sym13030379

[21] L. Iorio, "Local cosmological effects of the order of $H$ in the orbital motion of a binary system," Mon. Not. R. Astron. Soc. 429, 915-922, (2013).

[22] G. A. Krasinski, V.A. Brumberg, "Secular increase of astronomical unit from analysis of the major planet motions, and its interpretation," Celest. Mech. Dyn. Astr. 90, 267-288 (2004).

[23] M. Křížek, "Numerical experience with the finite speed of gravitational interaction," Math. Comput. Simulation 50, 237-245 (1999).

[24] M. Křižek, "Does a gravitational aberration contribute to the accelerated expansion of the Universe?" Comm. Comput. Phys. 5, 1030-1044 (2009).

[25] M. Křížek, "Dark energy and the anthropic principle," New Astronomy 17, 1-7 (2012).

[26] M. Kř́̌žek, Y. V. Dumin (eds.), Cosmology on small scales 2016, Proc. Internat. Conf., Inst. of Math., Prague, 2016.

[27] M. Křížek, Y. V. Dumin (eds.), Cosmology on small scales 2018, Proc. Internat. Conf., Inst. of Math., Prague, 2018.

[28] M. Křížek, Y. V. Dumin (eds.), Cosmology on small scales 2020, Proc. Internat. Conf., Inst. of Math., Prague, 2020.

[29] M. Kř́ížek, F. Křížek, L. Somer, Antigravity - its origin and manifestations, Lambert Acad. Publ., Saarbrücken, 2015. 
[30] M. Křížek, L. Somer, "Manifestations of dark energy in the Solar system," Gravit. Cosmol. 21, 58-71 (2015).

[31] L. R. Kump, J. F. Kastings, R. G. Crane, The Earth system, Prentice Hall, New Jersey, 1999.

[32] V. Lainey et al., "Resonance locking in giant planets indicated by the rapid orbital expansion of Titan," Nat. Astron. 4, 1053-1058 (2020).

[33] V. Lainey et al., "New tidal paradigm in giant planets supported rapid orbital expansion of Titan," 1-31, submitted in 2020.

[34] C. H. Lineweaver, D. Schwartzman, "Cosmic thermobiology," In: Origins (ed. J. Seckbach), Kluwer, Dordrecht, 233-248, 2003.

[35] G. J. F. MacDonald, "Tidal friction," Rev. Geophys. 2, 467-541 (1964).

[36] A. Maeder, "Scale invariant cosmology: Cosmological models and small scale effects," Proc. Internat. Conf. Cosmology on Small Scales 2016, Local Hubble Expansion and Selected Controversies in Cosmology (eds. M. Křížek, Y. V. Dumin), Inst. of Math., Prague, 41-64, 2016.

[37] A. Maeder, "An alternative to the $\Lambda$ CDM model: The case of scale invariance," Astrophys. J. 834, 194-209 (2017).

[38] A. Maeder, P. Bouvier, "Scale invariance, metrical connection and the motions of astronomical bodies," Astron. Astrophys. 73, 82-89 (1979).

[39] A. Maeder, V. G. Gueorguiev, "The scale-invariant vacuum (SIV) theory: A possible origin of dark matter and dark energy," Universe 46, 6, 1-33 (2020).

[40] A. Maeder, V. G. Gueorguiev, "On the relation of the lunar recession and the length-of-the-day," Astrophys. Space Sci. 366, 101 (2021).

[41] C. Martínez-Lombilla, I. Trujillo, J. H. Knapen, "Discovery of disc truncations above the galaxies mid-plane in Milky Way-like galaxies," Mon. Not. R. Astron. Soc. 483, 664-691 (2019).

[42] B. Mashhoon, N. Mobed, D. Singh, "Tidal dynamics in cosmological spacetimes," Classical Quant. Grav. 24, 5031-5046 (2007).

[43] D. Matson et al., "The thermal evolution and internal structure of Saturn's mid-sized icy satellites," In: Saturn from Cassini-Huygens (M. K. Dougherty, L. W. Esposito, S. M. Krimigis eds.), Springer, Dordrecht, 577-612, 2009.

[44] C. G. McVittie, "The mass-particle in expanding universe," Mon. Not. R. Astron. Soc. 93, 325-339 (1933).

[45] C. W. Misner, K. S. Thorne, J. A. Wheeler, Gravitation, 20th edition, W. H. Freeman, New York, 1997. 
[46] P. D. Noerdlinger, "Solar mass loss, the astronomical unit, and the scale of the Solar system," ArXiv: 0801.3807, 2008.

[47] E. Noether, "Invariante Variationsprobleme," Nachr. D. König. Gesellsch. D. Wiss. Zu Göttingen, Math.-phys. Klasse, Heft 2, 235-257 (1918).

[48] D. Obreschkow et al. (eds.), Galactic angular momentum, Proc. XXXth IAU General Assembly, Focus Meeting 6, Vienna, August, 2018.

[49] P. J. E. Peebles, Principles of physical cosmology, Princeton Univ. Press, New Jersey, 1993.

[50] W. R. Peltier, "History of Earth rotation." In: Treatise on Geophysics (ed. G. Schubert), vol. 9, Evolution of the Earth (vol. ed. D. Stevenson), Elsevier, Amsterdam, 2007.

[51] B. P. Pertsev, "On secular deceleration of the Earth's rotation," Izvestiya: Phys. Solid Earth 36, 218-222 (2000).

[52] Planck Collaboration, N. Aghanim et al., "Planck 2018 results. VI. Cosmological parameters," Astron. Astrophys. 641, A6 (2020).

[53] A. G. Riess et al., "Milky Way cepheid standards for measuring cosmic distances and application to Gaia DR2: Implications for the Hubble Constant," Astrophys. J. 861, Article ID 126 (2018).

[54] G. Risaliti, E. Lusso, "Cosmological constraints from the Hubble diagram of quasars at high redshifts," Nature Astronomy 3, 272-277 (2019).

[55] M. Scherf, H. Lammer, "Did Mars possess a dense atmosphere during the first $\sim 400$ million years?" Space Sci. Rev. 217, Article No. 2. (2021).

[56] B. W. Stiles et al., "The Cassini RADAR team: Determining Titan's spin state from Cassini RADAR images," Astron. J. 135, 1669-1680 (2008).

[57] T. C. van Flandern, "A determination of the rate of change of $g$," Mon. Not. R. Astron. Soc. 170, 333-342 (1975).

[58] A. Yahalom, "The effect of retardation on galactic rotation curves," J. Phys.: Conf. Ser. 1239, 012006 (2019).

[59] W. J. Zhang, Z. B. Li, Y. Lei, "Experimental measurements of growth patterns on fossil corals: Secular variation in ancient Earth-Sun distance," Chinese Sci. Bull. 55, 4010-4017 (2010). 